\title{
Task Performance vs. Learning Outcomes: A Study of a Tangible User Interface in the Classroom
}

\author{
Son Do-Lenh, Patrick Jermann, Sébastien Cuendet, \\ Guillaume Zufferey, and Pierre Dillenbourg \\ CRAFT, Ecole Polytechnique Fédérale de Lausanne (EPFL) \\ 1015 Lausanne, Switzerland \\ \{son.dolenh, patrick.jermann, sebastien.cuendet \\ guillaume.zufferey, pierre.dillenbourg\}@epfl.ch
}

\begin{abstract}
Tangible User Interfaces (TUIs) offer the potential to facilitate collaborative learning in new ways. This paper presents an empirical study that investigated the effects of a TUI in a classroom setting on task performance and learning outcomes. In the tangible condition, apprentices worked together around an interactive tabletop warehouse simulation using tangible inputs. In the paper condition, they performed the same activity with only paper and pens. Results showed that the tangible condition resulted in better task performance (more alternative solutions explored and better final solution) but did not affect learning outcomes, i.e. understanding of important concepts and applying them to a problem-solving question. We discuss reasons for this in terms of task structure and type, nature of tangible user interfaces and effective interaction requirements.
\end{abstract}

Key words: Tangible User Interface, Tabletop, Collaborative Learning, Augmented Reality, Technology-Enhanced Learning, Human Computer Interaction.

\section{Introduction}

With the fast development of technologies, educational scientists and practitioners have increased efforts to extend learning activities from traditional computers to other platforms, allowing richer experiences and more direct interactions. Among other paradigms, tangible user interfaces (TUI) have been actively researched recently. Very often, a TUI is augmented with visual information on a tabletop surface that works both as an input space and a display [28].

It is commonly believed that TUIs facilitate collaboration, especially collaborative learning scenarios, with learners not being constrained by obtrusive technologies and engaged in face-to-face, rather than mediated interactions. While it seems evident that TUIs provide new possibilities and enable many exciting activities $[4,15,29]$, it remains unclear as to whether students really benefit from using them in learning settings. 


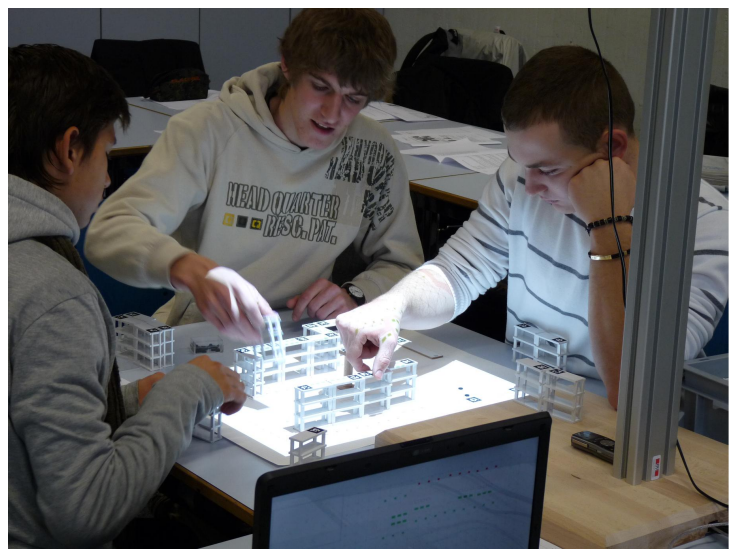

Fig. 1: Two apprentices building a warehouse model with the TinkerLamp.

In previous studies, we have observed qualitatively how apprentices at vocational schools collaborate around a TUI system called TinkerLamp (Figure 1) [13]. We also investigated the effects of this TUI when compared with a multitouch interface on learning outcomes in a controlled experiment [24]. While feedbacks from teachers and apprentices are positive with regards to the learning activities that we developed, we do not have formal proof for its benefits in a real classroom setting. In this paper, we set out to answer this question and hope the findings will give more insights into the future designs and usages of TUIs in realistic environments.

To this end, we conducted a study with groups of logistics apprentices studying in a classroom setting, under two conditions: a tangible condition and a baseline condition with traditional paper and pens. The given task was to collaboratively design a warehouse layout to learn concepts of different types of surfaces used to describe a warehouse.

\section{Related work}

Interactive tabletop computing (see e.g. [30,6]) is known for its support for fast and natural interactions. Among other things, people around a tabletop can interact with the system through various input styles, e.g. mouse or multi-mice [17], touch or hand gestures [6,23,10], or pens [18]. The horizontal nature of tabletop systems also affords users putting physical objects on the surface, and hence opens up the possibility for the intuitive manipulation of these tangibles (called Tangible User Interface) [28] which is the focus of our study.

The unique physical features of TUIs offer the potential to facilitate collaborative learning in new ways. They support building activities in which learners can interact directly with their hands by touching and manipulating objects. The benefits are manifold. 
First, manipulation of physical objects is useful for students' understanding since they are real and can be manipulated. The added value of sensori-motor experience that TUIs offer has been described in the literature [22], relying on the idea that they support an enactive mode of reasoning [3] and enable empirical abstractions of sensori-motor schemes [19].

Second, TUIs can enable more participation. Learning outcomes are generally dependent on students' level of participation in the activity. Learning benefits can be impaired when some group members dominate the whole activity [25]. TUIs hence play an important role to bring about more equitable participation thanks to simultaneous inputs. In [27], Stanton et. al. concluded that simultaneous inputs can promote more equitable interactions between children. In [22], the authors also discussed how they were able to promote engaging, active and playful learning by developing digitally augmented physical spaces.

Third, TUIs with the integration of external concrete inputs and the abstract augmented information may be an excellent means to present Multiple External Representations (MERs)[1]. The arguments put forward by this learning approach is that presenting learners with several instances of the same information at different levels of abstraction will act as a scaffold allowing them to understand the more abstract representation by observing how it is related to the more concrete one.

Fourth, most TUIs are used around a tabletop which provides a larger display area and helps group members to be better aware of each other's actions. This has been confirmed by several studies $[9,12]$. For example, through a study comparing groups of three people using mice versus a multi-touch table [12], Hornecker et. al. suggested that the affordances of direct touch input and body movements in tabletop condition resulted in a better awareness of intention and action than mice.

Although previous research has reported on how tangible tabletop technologies are adopted by school communities $[4,20,22,32,26]$, only a few applications are evaluated for learning outcomes $[14,31,8]$. Moreover, these studies have been mostly focused on young children, i.e. from primary-aged to about 14. Generally, the educational benefits of collaborative activities enabled by TUIs need further exploration and for different populations.

In this paper, we examine how effectively vocational apprentices, typically 17-20 years of age, study around a tabletop user interface using tangible objects as input. Before describing the study, we will first detail the educational context and our TUI system.

\section{Context}

\subsection{Vocational Training}

In countries like Germany, Austria and Switzerland, the prevailing organization of vocational training follows a dual approach: apprentices work three to four days per week in a company and spend one day per week in a vocational school. 
The dual model presents the advantage that apprentices gain experience by working in an authentic setting, and these experiences may be exploited in school courses. Likewise, the abstract concepts learned in school may also be referred to in their work environment.

The domain of interest was logistics, a profession that involves the storage and transportation of goods, the design of warehouses and transportation routes, as well as the management of inventories and information. Apprentices in logistics are students who usually have difficulties in reasoning skills. Consequently, it appears that many of them struggle to understand the logistics concepts presented at school.

Our field observations showed that the switching of context from the company (involving action) to the classroom (theory) potentially entails some difficulties for apprentices. Knowledge transfer from schools to the workplace is not automatic and would require specific training. Conversely, problems addressed in school appear as unspecific and inauthentic compared to the apprentices' daily practice. Bridging this abstraction gap is the focus of our research.

We developed a tangible tabletop environment, called the TinkerLamp. The main objective of this environment is to enable problem-solving activities which are as close as possible to the real context of a warehouse to help apprentices overcome the lack of abstraction skills.

\subsection{TinkerLamp: a tangible tabletop environment}

The TinkerLamp system (Figure 2) is currently used in several vocational schools [33]. Apprentices and teachers interact with the TinkerLamp through a tangible user interface, consisting of two interaction modalities.

First, it involves using a small-scale model of a warehouse with miniature plastic shelves, loading docks, offices, etc. to build a physical warehouse layout (Figure 2b). Each element of the small-scale warehouse is tagged with a fiducial marker that enables automatic camera object recognition. This warehouse model is augmented with visual feedback and information through a projector above the table. The simplest augmentation is the drawing of navigation nodes around each shelf. When two shelves are placed too close, the navigation nodes turn red, showing that there is not enough space for a forklift to work in the alley between these shelves. It is therefore possible for apprentices to find the ideal alley width by trial and error.

Second, users use a paper-based interface (called TinkerSheet, Figure 2) to change parameters of the system or to run and control a simulation on top of the small-scale model. The simulation displays real-time information, e.g. various statistics about the warehouse inventory or storage management strategies. TinkerSheets are a set of paper pieces, used to set simulation parameters and visualize outputs on the same physical space which is used to build the warehouse model. Users can interact with a sheet in a couple of ways, using either physical tokens (e.g. magnets) or a pen by drawing a circle on an input area. 


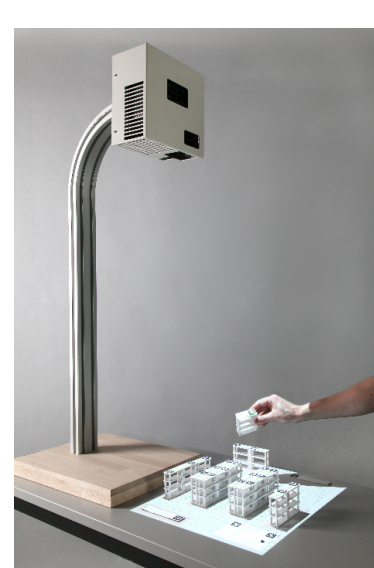

(a)

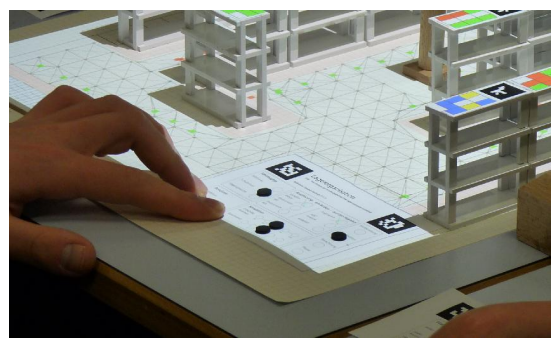

(c)

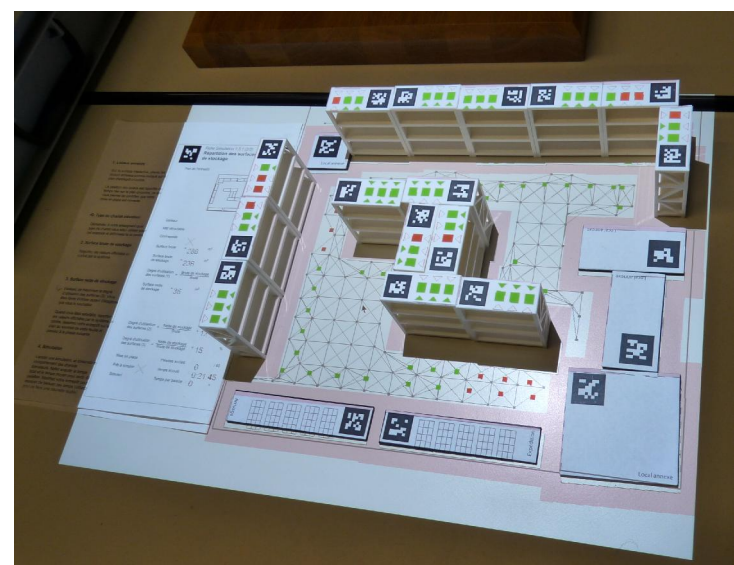

(b)

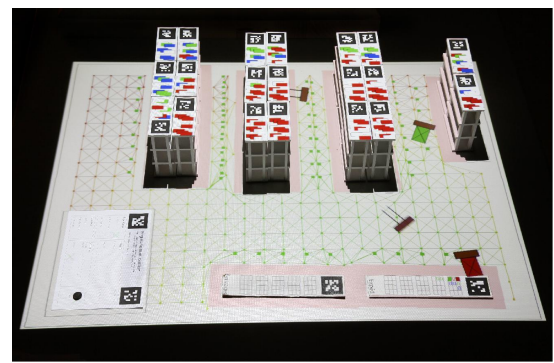

(d)

Fig. 2: Tinker systems: a) TinkerLamp. b)Small-scale model with a TinkerSheet to the left. c)Use TinkerSheet to change system's parameters. d) Simulation of the warehouse

\section{Research hypotheses}

We are interested in a problem-solving task, which is the design of a warehouse. This task is a common learning task in logistics classes. It requires the apprentices to explore the warehouse surfaces and problem-solve the placement of different warehouse elements (docks, storage shelves, administration offices, etc.) by taking into account various constraints. Drawing with paper and pens has been the traditional method to tackle this type of problems during the logistics apprenticeship. The aim of our contribution is to examine the impact of TUIs on learning outcomes compared to this method.

We conducted an experimental study in a classroom setting. Groups of apprentices in the experimental condition were provided with TinkerLamps and tangible inputs. The groups in the control condition were not provided with them, rather working together in a traditional way using paper and pens. 
We expected that the physical and social natures of TUIs would result in both better task performance and better learning outcomes. More specifically, we tested the following hypotheses.

1. Tangible interface leads to better task performance ( more alternative solutions, better final designs) than the traditional paper-and-pen approach.

2. Tangible interface leads to more positive learning outcomes (understanding and applying concepts in other situations better ) than the traditional paperand-pen approach.

Our aim with TinkerLamp is to develop a system that can be used in authentic settings without researcher's presence. Consequently, our study took place in a classroom and also involved teachers. We would like to explore how the TUI works in a setting as close to reality as possible.

\section{Experiment design}

\subsection{Participants}

Four classes with a total of 61 apprentices participated in the study. Due to the logisctics domain characteristics, most of them are male (56 out of 61 ). The two experimental conditions were: (1) the tangible condition (hereafter called TAN condition, two classes of 15 students), in which the students collaborated around TinkerLamps; (2) the paper condition (PAP, a class of 15 students, a class of 16), in which the activity involved students drawing on paper with pens (Figure 3). There were two teachers, each managing a different class of 15 or 16 students in each of the conditions.

\subsection{Learning task}

The objective of the learning task was to teach different types of surfaces that are used to design a warehouse: raw surface, raw storage surface and net storage surface. The raw surface is simply the surface of the whole warehouse. The raw storage surface is the raw surface minus annex rooms (offices, technical rooms, docks, etc.) The net storage surface is the raw storage surface minus the corridors where the forklifts travel on. The apprentices were required to understand what constitutes each type of surfaces and their impact on work effciency.

The task was to design a warehouse layout through several steps guided by an instruction paper, with the help from the teacher. At the beginning, groups of four apprentices, sitting around a table in the classroom, were instructed to design a warehouse with only ten shelves (which correspond to a very low usage of the warehouse surface), and compute the surfaces and the ratios. The next step required each group to try to augment the percentage of net storage surface by maximizing the number of shelves in the warehouse. Once they were done, they were asked to compute the surfaces and ratios again.

In the paper condition, they drew the warehouse on paper using pens, erasers and rulers. In the tangible condition, the group built the warehouse by placing 


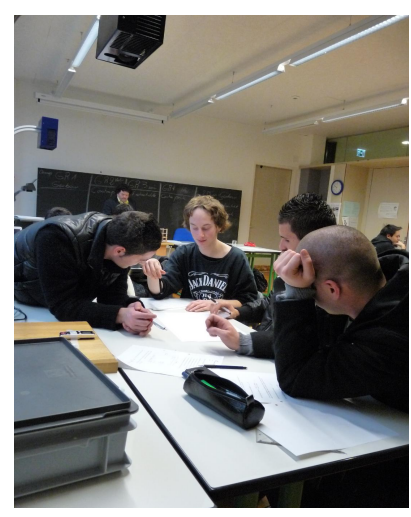

(a)

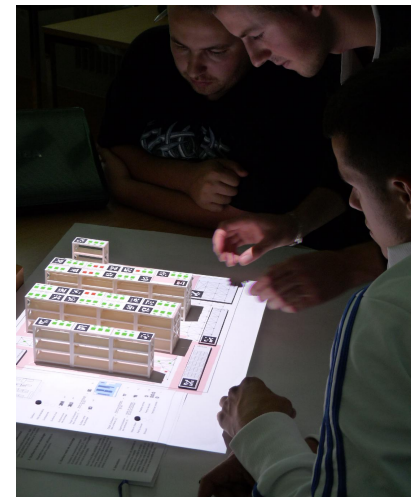

(c)

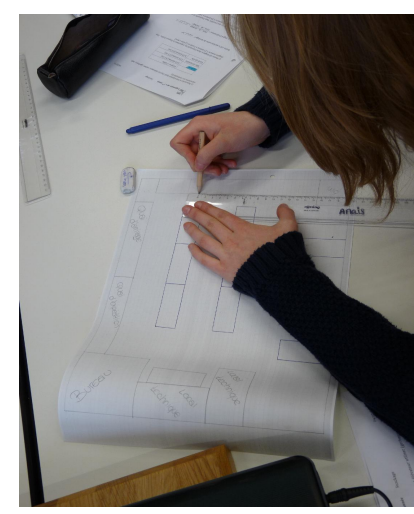

(b)

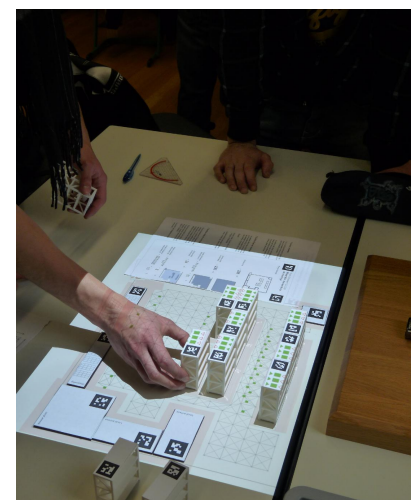

(d)

Fig. 3: Two experimental conditions: a,b) Paper condition. c,d) Tangible condition.

tangible shelves under the TinkerLamp. The surface areas would also be automatically calculated and projected on the table by the system. The apprentices only needed to note down those statistics, instead of computing them. They also ran a simulation on the layouts they had built to see how the work efficiency are affected.

After completing the task, each group was asked to deduce some conclusions from their results. They would need to reflect on the layouts they had built, on the concepts of surfaces, e.g. see how different constraints affect surfaces areas and ratios, etc. At the end of the session, the teacher organized a debriefing session where the conclusions of each group were discussed.

The structure of the activity was a result of a participatory design process between researchers and the teachers participating in the study. While two conditions differ in a couple of ways due to the nature of the interfaces, the steps and contents to be transfered across two conditions are ensured to be very similar. 


\subsection{Procedure}

\begin{tabular}{|c|c|c|c|c|}
\hline $\begin{array}{c}\text { Pre-test } \\
(10 \mathrm{mins})\end{array}$ & $\begin{array}{c}\text { Introduction lecture } \\
(30 \mathrm{mins})\end{array}$ & $\begin{array}{c}\text { Group activity } \\
(60 \mathrm{mins})\end{array}$ & $\begin{array}{l}\text { Post-test } \\
(20 \mathrm{mins})\end{array}$ \\
\hline
\end{tabular}

Fig. 4: Four phases in the study

Figure 4 shows an overview of the four phases of the study. First, apprentices did a brief individual pre-test in 10 minutes. It is a simple set of five math questions, testing the apprentices of their basic knowledge of reasoning and math calculations.

After the pre-test, a typical session involved three steps: introduction lecture (30 mins), group activity (60 mins) and class debriefing (30 mins). During the lecture, the teacher gave the apprentices introduction about concepts that they were to learn.

The class was then divided into four groups to explore more about those concepts. There were paper instructions for apprentices as to how to proceed with the task. As presented in section 5.2, the groups had to collaborate to build warehouse models and discover how the concepts they had been introduced would be applied in these particular models.

The teacher toured around the room to respond to help requests. He used the last 30 minutes to recap the main points of the session. An individual post-test was administered at the end of the session to measure learning gains.

\subsection{Usability issues}

Prior to this study, a series of usability tests had been conducted and major problems had been corrected. The system was also used during the last two years by the same teachers with other classes. Moreover, during the study, we noted that the apprentices encountered no problem understanding and using the system. Even though they were using it for the first time, they got quickly involved in the activity without much time asking interface-related questions.

\subsection{Dependent variables}

Four dependent variables were used in this paper: (1) number of alternative solutions explored. (2) the quality of the final layout. (3) understanding score. (4) problem-solving score.

Task performance was determined by two variables (1) and (2). The number of alternative solutions is the number of warehouse layouts that the group tried out during the activity and was counted based on video and audio recordings. The quality of the final layout is computed based on the number of shelves that 
the apprentices succesfully placed in the warehouse. This variable implies that the warehouse will be able to store more goods with more shelves.

Learning performance was determined by two variables (3) and (4), both through the post-test. The post-test consisted of two parts, graded separately. The first part checks the understanding of the surface concepts, asking apprentices to compare different warehouses according to some aspect, e.g. to choose the one with the largest net storage surface. It consists of 12 multiple-choice questions, each of which included four possibilities with only one correct answer, and one to state "I don't know the answer". One correct answer corresponds to a point in the understanding score. There is no penalty for choosing a wrong answer.

In the second part, the apprentices had to answer an open-ended question with a realistic warehouse problem. They were told to consider a warehouse design and were asked to propose to the director how to maximize the efficiency of the warehouse. We consider it a problem-solving process since there are multiple possible solutions and the answer may be evaluated by multiple criteria. It involves, for example, taking into account the trade-off between two constraints: having as many shelves as possible and maintaining enough corridor space for forklifts to move around.

As the post-test is carried out with paper and pencil, it can be argued that there is a bias towards the paper condition. In fact, the first part of the post-test only involves multiple-choice questions and requires cognitive reasoning to solve, instead of performing a drawing activity. Paper and pencil are just a means to answer those questions, and hence will unlikely produce any effects on the results. The apprentices could have been asked to answer those same questions with a computer keyboard. The second part score was coded in a way to test only the understanding of the apprentices, and not the drawing skills. Moreover, testing on paper is also the traditional way of grading in these classes, and we were tempted to use the same measurement to judge the apprentices.

\section{Results}

\subsection{Task performance}

Alternative solutions. It is evident that in the course of collaboration, apprentices in the TAN condition explored more alternative layouts than those in the paper condition. Over the activity, groups who used the tangible interface completed 4.6 layouts on average (median =4), which is higher than those who were in the paper condition ( mean $=2.5$, median $=2$ ). This divergence in number of alternative layouts was significant, confirmed by a Wilcox-test, $W(14)=8.0, p<.01$.

Quality of final solution. The apprentices in the TAN condition designed better final solutions compared to the PAP condition. A t-test revealed a significant 
higher number of shelves being placed in the final layout in the tangible condition $(t(14)=2.36, p<.05)$. They managed to use more space in the warehouse, succesfully placing 18.0 shelves on average in the final warehouse, compared to 15.1 shelves by those using paper and pens.

\subsection{Learning outcomes}

Understanding score. We found that the interface apprentices had used during the study had no signicant effect on the understanding score. A Wilcoxon test found no significant difference between individual scores in the two conditions, $W(59)=506 ; p>.05$, though paper groups gained higher on average (M $=7.84 \mathrm{vs} \mathrm{M}=7.43$ for paper and tangible groups respectively).

We replicated the above test using another coding of the data, with which apprentices are penalized a point of -1 if they answer the question wrong and 0 if they mark "I don't know the answer". The results turned out to be no different. When using paper and tangible interface, the apprentices respectively scored on average 3.8 points and 3.1 points; not a significant difference: $W(59)=504 ; p>$ .05 .

Problem-solving score. We graded the open-ended question in different aspects: whether the apprentices successfully augmented the net storage surface in the warehouse, how detailedly and correctly they justified their propositions, whether they remembered to put shelves in pair (an important concept when designing a warehouse), whether their propositions ensured enough alley space for forklifts' movements and manipulations, etc.

The final score is the average of all those partial scores. Apprentices in the PAP condition got an average score of 5.55, as opposed to 5.13 in the TAN condition, not a significant difference $(W(59)=520, p>.05)$.

\section{Discussion}

\subsection{Task performance}

As expected, our findings showed that the task performance (number of alternative solutions explored and quality of the final solution) in the tangible condition is higher than that in the paper condition. This can be explained, first, by the direct interaction mechanism. Obviously, grasping a tangible shelf and placing it on the table to create the model is faster. Modifying the model is also facilitated by the fact that tangibles leave no traces behind. Apprentices could simply move shelves to another position, as opposed to erasing and re-drawing the layout with paper and pens. Simultanous actions also speed up the process. Design iterations were therefore done quickly which saved time for apprentices to try out other possible options.

Second, the task performance difference may concern the difference in information perception from the design space. The level of correspondence between 
a real warehouse and the design representation differs across the two conditions. The tangible small-scale model gives a concrete and 3D physical representation of a warehouse, in comparison with the more abstract representation offered by the $2 \mathrm{D}$ drawings in the paper condition. With difficulties in reasoning and abstraction skills, apprentices encountered problems in expressing their ideas into drawings [13]. Few of them were correct in terms of scaling. Hence, the tangible interface may have an advantage in this respect. An issue that can be further explored is the use of 2D PostIt-like paper pieces in the activity. It would be interesting to observe how the apprentices would exploit these objects instead of drawing as the $2 \mathrm{D}$ representation.

Third, we believe the different strategies adopted across the two condtions also play a role. Our observations revealed that those in paper condition tended to discuss about the solution first, then execute a perfect plan on paper. This differs with those in the tangible condition. They typically followed a bottom-up approach, incrementally added shelves on the table and tweaked their positions to choose the best layout. This strategy, made possible by the TUI, enables the apprentices to test small variations of the solution and come up with better ones.

\subsection{Learning outcomes}

Contrary to our hypotheses, there is no evidence that the tangible condition had more effects than the paper condition on either the understanding or the problem-solving score. We discuss reasons for this in terms of task structure and type, physical properties of TUIs and effective interaction requirements.

The first dimension that we saw as important is how loosely or tightly structured the task was. In our study, it can be argued that the activity is not really a "group task" according to the definition of Cohen [5], in the sense that it could have been carried out by individuals separately (although roles did emerge). The type of the activity is another factor. The group activity is somewhat structured. That means that the apprentices did not need to exchange and discuss their own understanding to other group members and hence the collaboration that the tangible interface offers might not have been taken advantage. This finding is consistent with some of the literature in traditional collaborative learning [5]. Moreover, it is worth noting that the open-ended question was an ill-structured problem which takes time to be learned and the skills needed for it had not been trained during the activity.

The second dimension is the nature of TUIs. As much as it contributed to improve the task performance, this nature may have a detrimental effect on learning. First, the warehouse layouts were accomplished "too fast". It may have resulted in a less concentrated and intensive cognitive effort to make sense of the solutions and to understand deeper. Besides, previous studies have suggested that the physical properties of tangible tabletops and their support for parallel work can also lead to limited reciprocity and mutual understanding $[16,7,11]$. In [7], it has been found that students using a tangible interface gained fewer in test scores because group members tended to perform individual actions without discussing much with the others. 
Another possible explanation for the lack of signicant effect is that students needed both social and cognitive skills for effective interaction [2]. While it is true that the tangible user interface encouraged the generation of more alternative options, the required skills (e.g. the social skills to modify and converge different viewpoints, or the cognitive skills to set up and test hypotheses when play around with various layouts, etc.) to use them are a real challenge for the logistics apprentices. Although we did not assess collaboration quality, we argue that their collaboration might not have been effective enough to bring about more understanding.

\subsection{General discussion}

Our study is different from other related works in several aspects. First, we focus on a different student population, vocational apprentices to be specific, instead of younger children. Second, we wanted to look at the effects tangible interface have on learning outcomes, instead of only collaboration quality, strategy, conversations or task performance. Third, we ran our study in a classroom setting to ensure a realistic learning scenario. The study is also seen as complementary to our previous studies: qualitative analyses of how students collaborate around a tangible tabletop [13], and a controlled experiment comparing tangible and multi-touch interface in a lab setting [24]. We believe that by having a real scenario, the effects of artificial experiment factors on observed behaviours are reduced.

By focusing on these different variables and settings, we have found interesting results. Tangible tabletops, despite all of its potential, may require special designs when integrated in classrooms. While our findings about the better task performance measures in the tangible condition are in line with other studies $[16,21,14]$, the results also suggested its learning outcomes are not necessarily better than the traditional paper-and-pens way of teaching and studying. In fact, a recent experiment also found a similar result[20].

We argue that the extent to which tangible user interfaces can improve learning is largely dependent on educational scenarios, involving different actors (the nature of the task, students's skills, teachers, group size, etc.), rather than an inherent characteristic of the technology. While TUI may have many benefits as stated at the beginning, when it becomes a part of classroom practice, many factors may well intefere in a complex way and contribute to the final effects.

\section{Conclusion}

We have presented our experiment in a classroom setting, comparing a tangible user interface against the traditional way of studying using paper and pens in terms of task performance and learning outcomes. As expected, apprentices who used the TUI scored better in task performance criteria. They explored more alternative solutions during the learning task and designed warehouse layouts with higher quality. However, we found no evidence of significant effects on 
learning outcomes. We discussed reasons for this in terms of the nature of the task, the physical properties of TUIs, and skills required for effective group learning.

It is arguable that more than a single factor affected the learning process in our study, and the inherent characteristics of tangible computing technology does not necessarily bring about more positive outcomes in classroom settings. Our findings have implications both for educational practitioners who are deploying similar technologies in classrooms and also for the designers of future tangible tabletops.

Acknowledgments. This research is part of "Dual-T: technologies for Vocational Training", a leading house funded by the Swiss Federal Office for Professional Education and Technology. We would like to thank the teachers and students from the vocational school for their enthusiasm to support our work, especially to Jacques Kurzo, Olivier Vonlanthen, André Ryser and the participants of our current study. We would like to thank Olivier Guedat for technical support during the study.

\section{References}

1. S. Ainsworth. Deft: a conceptual framework for considering learning with multiple representations. Journal of Learning and Instruction, 2006.

2. D. Barnes and F. Todd. Communication and learning in small groups. London: Routledge \& Kegan Paul, 1977.

3. J. S. Bruner. Toward a Theory of Instruction. Cambridge: Belknap Press, 1966.

4. X. Cao, S. E. Lindley, J. Helmes, and A. Sellen. Telling the whole story: anticipation, inspiration and reputation in a field deployment of telltable. In $C S C W$ '10: Proceedings of the 2010 ACM conference on Computer supported cooperative work, pages 251-260, New York, NY, USA, 2010. ACM.

5. E. G. Cohen. Restructuring the classroom: Conditions for productive small groups. Review of Educational Research, 64(1):1-35, 1994.

6. P. Dietz and D. Leigh. Diamondtouch: A multi-user touch technology. ACM Symposium on User Interface Software and Technology (UIST'01), pages 219-226, - 2001-.

7. S. Do-Lenh, F. Kaplan, and P. Dillenbourg. Paper-based concept map: the effects of tabletop on an expressive collaborative learning task. In BCS HCI '09: Proceedings of the 2009 British Computer Society Conference on Human-Computer Interaction, pages 149-158, Swinton, UK, UK, 2009. British Computer Society.

8. Y. Fernaeus and J. Tholander. Finding design qualities in a tangible programming space. In CHI '06: Proceedings of the SIGCHI conference on Human Factors in computing systems, pages 447-456, New York, NY, USA, 2006. ACM Press.

9. V. Ha, K. M. Inkpen, R. L. Mandryk, and T. Whalen. Direct intentions: the effects of input devices on collaboration around a tabletop display. Horizontal Interactive Human-Computer Systems, 2006. TableTop 2006. First IEEE International Workshop on, pages 8 pp.+, 2006.

10. J. Y. Han. Low-cost multi-touch sensing through frustrated total internal reflection. Proceedings of the 18th annual ACM symposium on User interface software and technology, pages 115-118, 2005. 1095054 115-118. 
11. A. Harris, J. Rick, V. Bonnett, N. Yuill, R. Fleck, P. Marshall, and Y. Rogers. Around the table: are multiple-touch surfaces better than single-touch for children's collaborative interactions? In CSCL'09: Proceedings of the 9th international conference on Computer supported collaborative learning, pages 335-344. International Society of the Learning Sciences, 2009.

12. E. Hornecker, P. Marshall, N. S. Dalton, and Y. Rogers. Collaboration and interference: awareness with mice or touch input. CSCW' 08 : Proceedings of the ACM 2008 conference on Computer supported cooperative work, pages 167-176, 2008.

13. P. Jermann, G. Zufferey, and P. Dillenbourg. Tinkering or sketching: Apprentices' use of tangibles and drawings to solve design problems. In LNCS, Times of Convergence. Technologies Across Learning Contexts, pages 167-178, 2008.

14. M. Khandelwal and A. Mazalek. Teaching table: a tangible mentor for pre-k math education. In TEI '0\%: Proceedings of the 1st international conference on Tangible and embedded interaction, pages 191-194, New York, NY, USA, 2007. ACM.

15. A. Manches, C. O'Malley, and S. Benford. Physical manipulation: evaluating the potential for tangible designs. In TEI '09: Proceedings of the 3rd International Conference on Tangible and Embedded Interaction, pages 77-84, New York, NY, USA, 2009. ACM.

16. P. Marshall, E. Hornecker, R. Morris, N. Sheep Dalton, and Y. Rogers. When the fingers do the talking: A study of group participation with varying constraints to a tabletop interface. In 3rd IEEE International Workshop on Horizontal Interactive Human Computer Systems, 2008. TABLETOP 2008., pages 33-40, 2008.

17. C. Müller-Tomfelde and C. Schremmer. Touchers and mousers: commonalities and differences in co-located collaboration with multiple input devices. CHI '08: Proceeding of the twenty-sixth annual SIGCHI conference on Human factors in computing systems, pages 1149-1152, 2008 .

18. M. A. Nacenta, D. Pinelle, D. Stuckel, and C. Gutwin. The effects of interaction technique on coordination in tabletop groupware. GI '07: Proceedings of Graphics Interface 200\%, pages 191-198, 2007.

19. J. Piaget. The future of developmental child psychology. Journal of Youth and Adolescence, 3:87-93, 1974.

20. A. M. Piper and J. D. Hollan. Tabletop displays for small group study: affordances of paper and digital materials. In CHI '09: Proceedings of the 27th international conference on Human factors in computing systems, pages 1227-1236, New York, NY, USA, 2009. ACM.

21. A. M. Piper, E. O'Brien, M. R. Morris, and T. Winograd. Sides: a cooperative tabletop computer game for social skills development. In $C S C W$ '06: Proceedings of the 2006 20th anniversary conference on Computer supported cooperative work, pages 1-10, New York, NY, USA, 2006. ACM.

22. S. Price and Y. Rogers. Let's get physical: the learning benefits of interacting in digitally augmented physical spaces. Comput. Educ., 43(1-2):137-151, 2004.

23. J. Rekimoto. Smartskin: an infrastructure for freehand manipulation on interactive surfaces. In Proceedings of the SIGCHI Conference on Human Factors in Computing Systems (CHI'02), pages 113-120, 2002.

24. B. Schneider, P. Jermann, G. Zufferey, and P. Dillenbourg. Benefits of a tangible interface for collaborative learning and interaction. IEEE Transactions on Learning Technologies, under revision.

25. Slavin. Cooperative learning: theory, research and practice. 2nd Ed. Allyn \& Bacon, 1995. 
26. D. Stanton, V. Bayon, H. Neale, A. Ghali, S. Benford, S. Cobb, R. Ingram, C. O'Malley, J. Wilson, and T. Pridmore. Classroom collaboration in the design of tangible interfaces for storytelling. In $C H I$ '01: Proceedings of the SIGCHI conference on Human factors in computing systems, pages 482-489, New York, NY, USA, 2001. ACM.

27. D. Stanton, H. Neale, and V. Bayon. Interfaces to support children's co-present collaboration: multiple mice and tangible technologies. In CSCL '02: Proceedings of the Conference on Computer Support for Collaborative Learning, pages 342-351. International Society of the Learning Sciences, 2002.

28. B. Ullmer and H. Ishii. The metadesk: models and prototypes for tangible user interfaces. ACM Symposium on User interface Software and Technology, (UIST97), pages 223-232, 1997.

29. J. Underkoffler and H. Ishii. Illuminating light: a casual optics workbench. In $C H I$ '99: CHI '99 extended abstracts on Human factors in computing systems, pages 5-6, New York, NY, USA, 1999. ACM.

30. P. Wellner. Interacting with paper on the digital desk. Communications of the ACM, 36(7):87-96, 1993.

31. O. Zuckerman, S. Arida, and M. Resnick. Extending tangible interfaces for education: digital montessori-inspired manipulatives. In CHI '05: Proceedings of the SIGCHI conference on Human factors in computing systems, pages 859-868, New York, NY, USA, 2005. ACM Press.

32. O. Zuckerman and M. Resnick. System blocks: A physical interface for system dynamics simulation. In Proceedings of CHI '03, 2003.

33. G. Zufferey, P. Jermann, S. Do-Lenh, and P. Dillenbourg. Using augmentations as bridges from concrete to abstract representations. In BCS HCI '09: Proceedings of the 2009 British Computer Society Conference on Human-Computer Interaction, pages 130-139, Swinton, UK, 2009. British Computer Society. 